\title{
ON WEIGHTED SOBOLEV INTERPOLATION INEQUALITIES
}

\author{
SENG-KEE CHUA \\ (Communicated by Andrew M. Bruckner)
}

\begin{abstract}
We obtain some weighted Sobolev interpolation inequalities on $\mathbb{R}^{n}$ and domains satisfying the Boman chain condition for doubling weights satisfying a weighted Poincaré inequality.
\end{abstract}

\section{INTRODUCTION}

Recently, there has been a significant number of papers on weighted Sobolev interpolation inequalities, for example, Brown and Hilton [3-5], Gutierrez and Wheeden [18], and Chua [9]. In this paper, we will study weighted Sobolev interpolation inequalities with weights satisfying the following inequality:

$$
\left\|f-f_{Q}\right\|_{L_{w}^{q}(Q)} \leq A(Q)\|\nabla f\|_{L_{v}^{p}(Q)}
$$

as in [18] where $f_{Q}=\int_{Q} f d x /|Q|$. Let us note that some sufficient conditions have been obtained for (1.1); see [27], [26], or [7].

Definition 1.1 [19]. An open set $\mathscr{D}$ in $\mathbb{R}^{n}$ is said to be a member of $\mathscr{F}(\sigma, N)$, $\sigma \geq 1, N \geq 1$, if there exists a covering $W$ of $\mathscr{D}$ consisting of cubes such that:

(i)

$$
\sum_{Q \in W} \chi_{\sigma Q}(x) \leq N \chi_{\mathscr{D}}(x) \quad \forall x \in \mathbb{R}^{n} .
$$

(ii) There is a 'central cube' $Q_{0} \in W$ that can be connected with every cube $Q \in W$ by a finite chain of cubes $Q_{0}, Q_{1}, \ldots, Q_{k(Q)}=Q$ from $W$ such that $Q \subset N Q_{j}$ for $j=0,1, \ldots, k(Q)$. Moreover, $Q_{j} \cap Q_{j+1}$ contains a cube $R_{j}$ such that $Q_{j} \cup Q_{j+1} \subset N R_{j}$.

We say that $\mathscr{D}$ satisfies the Boman chain condition if $\mathscr{D} \in \mathscr{F}(\sigma, N)$ for some $N, \sigma \geq 1$. There are many types of domains that satisfy the Boman chain condition, for example, balls, cubes, and John domains (see [19]). Moreover, it is easy to check that bounded $(\varepsilon, \infty)$ domains (see [20] or [9] for the definition) satisfy the Boman chain condition. Hence, so do bounded Lipschitz domains. In what follows, $Q$ is always a cube and $l(Q)$ will be its edgelength. If $1<p<$

Received by the editors July 23, 1992.

1991 Mathematics Subject Classification. Primary 26D10, 46E35.

Key words and phrases. Poincaré inequalities, doubling weights, $A_{p}$ weights, domains satisfying the Boman chain condition, Sobolev interpolation inequalities. 
$\infty, p^{\prime}$ will denote $p /(p-1)$. By a weight $w$, we mean a nonnegative locally integrable function on $\mathbb{R}^{n}$. By abusing notation, we will also write $w$ for the measure induced by $w$. Sometimes we write $d w$ to denote $w d x$. We say that $w$ is doubling if $w(2 Q) \leq C w(Q)$ for every cube $Q$, where $2 Q$ denotes the cube with the same center as $Q$ and twice its edgelength. By $w \in A_{p}$, we mean $w$ satisfies the Muckenhoupt $A_{p}$ condition, i.e.,

$$
\frac{1}{|Q|}\left(\int_{Q} w d x\right)^{1 / p}\left(\int_{Q} w^{-1 /(p-1)} d x\right)^{1 / p^{\prime}} \leq C \quad \text { when } 1<p<\infty
$$

and

$$
\frac{1}{|Q|} \int_{Q} w(x) d x \leq C \underset{x \in Q}{\operatorname{essinf}} w(x) \text { when } p=1,
$$

for all cubes $Q$ in $\mathbb{R}^{n}$. Note that $w$ is doubling when it is in $A_{p}$.

Let $\mathscr{D}$ be an open set in $\mathbb{R}^{n}$. If $\alpha$ is a multi-index, $\alpha=\left(\alpha_{1}, \alpha_{2}, \ldots, \alpha_{n}\right) \in$ $\mathbb{Z}_{+}^{n}$, we will denote $\sum_{j=1}^{n} \alpha_{j}$ by $|\alpha|$ and $D^{\alpha}=\left(\partial / \partial x_{1}\right)^{\alpha_{1}} \cdots\left(\partial / \partial x_{n}\right)^{\alpha_{n}}$. We denote by $\nabla$ the vector $\left(\partial / \partial x_{1}, \partial / \partial x_{2}, \ldots, \partial / \partial x_{n}\right)$ and by $\nabla^{m}$ the vector of all possible $m$ th-order derivatives for $m \in \mathbb{N}$. A locally integrable function $f$ on $\mathscr{D}$ (we will write $f \in L_{\text {loc }}^{1}(\mathscr{D})$ ) has a weak derivative of order $\alpha$ if there is a locally integrable function (denoted by $D^{\alpha} f$ ) such that

$$
\int_{\mathscr{D}} f\left(D^{\alpha} \varphi\right) d x=(-1)^{|\alpha|} \int_{\mathscr{D}}\left(D^{\alpha} f\right) \varphi d x
$$

for all $C^{\infty}$ functions $\varphi$ with compact support in $\mathscr{D}$ (we will write $\varphi \in$ $\left.C_{0}^{\infty}(\mathscr{D})\right)$.

For $1 \leq p<\infty, k \in \mathbb{N}$, and any weight $w, L_{w, k}^{p}(\mathscr{D})$ and $E_{w, k}^{p}(\mathscr{D})$ are the spaces of functions having weak derivatives of all orders $\alpha,|\alpha| \leq k$, and satisfying

$$
\|f\|_{L_{w, k}^{p}(\mathscr{D})}=\sum_{0 \leq|\alpha| \leq k}\left\|D^{\alpha} f\right\|_{L_{w}^{p}(\mathscr{D})}=\sum_{0 \leq|\alpha| \leq k}\left(\int_{\mathscr{D}}\left|D^{\alpha} f\right|^{p} d w\right)^{1 / p}<\infty
$$

and

$$
\|f\|_{E_{w, k}^{p}(\mathscr{D})}=\sum_{|\alpha|=k}\left\|D^{\alpha} f\right\|_{L_{w}^{p}(\mathscr{D})}<\infty,
$$

respectively. Moreover, in the case when $w \equiv 1$, we will denote $L_{w, k}^{p}(\mathscr{D})$ and $E_{w, k}^{p}(\mathscr{D})$ by $L_{k}^{p}(\mathscr{D})$ and $E_{k}^{p}(\mathscr{D})$, respectively. Finally, let $\Lambda^{k}(\mathscr{D})$ be the collection of all functions $f$ on $\mathscr{D}$ such that all its weak derivatives of order $\leq k$ exist.

We will prove that

Theorem 1.2. Let $\mathscr{D} \in \mathscr{F}(\sigma, N)$, and let $W$ be a covering of $\mathscr{D}$ satisfying the Boman chain condition. Let $1 \leq p \leq q<\infty$. If $v$ is a weight and $w$ is a doubling weight such that (1.1) holds for all $Q \in W$ and $f \in \Lambda^{1}(\mathscr{D})$, then

$$
\begin{aligned}
\|\nabla f\|_{L_{w}^{q}(\mathscr{D})} \leq & C w\left(Q_{0}\right)^{1 / q} l\left(Q_{0}\right)^{-n}\left(l\left(Q_{0}\right)^{-1}\|f\|_{L^{1}\left(Q_{0}\right)}+l\left(Q_{0}\right)\left\|\nabla^{2} f\right\|_{L^{1}\left(Q_{0}\right)}\right) \\
& +C A_{0}\left\|\nabla^{2} f\right\|_{L_{v}^{p}(\mathscr{D})}
\end{aligned}
$$

for all $f \in \Lambda^{2}(\mathscr{D})$ where $A_{0}=\sup _{Q \in W} A(Q), Q_{0}$ is the 'central' cube in $W$, and $C$ is independent of $f$ and $v$. 
Theorem 1.3. Let $1 \leq p \leq q<\infty$. Suppose that $v$ is a weight and $w$ is $a$ doubling weight such that

$$
\left\|f-f_{Q}\right\|_{L_{w}^{q}(Q)} \leq C_{0} w(Q)^{1 / q} v^{\prime}(Q)^{1 / p^{\prime}} l(Q)^{-n+1}\|\nabla f\|_{L_{v}^{p}(Q)}
$$

for all cubes $Q$ and $f \in \Lambda^{1}\left(\mathbb{R}^{n}\right)$ where $v^{\prime}=v^{-1 /(p-1)}\left(v^{\prime}(Q)^{1 / p^{\prime}}=\right.$

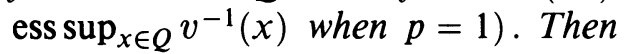

$$
\begin{aligned}
\left\|\nabla^{k} f\right\|_{L_{w}^{q}(Q) \leq} & C w(Q)^{1 / q} l(Q)^{-n-k}\|f\|_{L^{1}(Q)} \\
& +C w(Q)^{1 / q} v^{\prime}(Q)^{1 / p^{\prime}} l(Q)^{-n+1}\left\|\nabla^{k+1} f\right\|_{L_{v}^{p}(Q)}
\end{aligned}
$$

for all cubes $Q$ and $f \in \Lambda^{k+1}\left(\mathbb{R}^{n}\right)$ where $C$ is independent of $f$.

Moreover, if $\left\|\nabla^{k+1} f\right\|_{L_{v}^{p}\left(\mathbb{R}^{n}\right)} \neq 0$ and there exist $a<1, b>(1-k) / 2$, $1 \leq p_{0} \leq q$, and weight $v_{0}$ such that

$$
l(Q)^{(2 b-1-n)} w(Q)^{1 / q} v_{0}^{\prime}(Q)^{1 / p_{0}^{\prime}}+l(Q)^{(2 a-1-n)} w(Q)^{1 / q} v^{\prime}(Q)^{1 / p^{\prime}} \leq C
$$

for all cubes $Q$, then

$$
\left\|\nabla^{k} f\right\|_{L_{w}^{q}\left(\mathbb{R}^{n}\right)} \leq C\|f\|_{L_{v_{0}}^{p_{0}}\left(\mathbb{R}^{n}\right)}^{1-(2 b+k-1) /(1+k+2(b-a))}\left\|\nabla^{k+1} f\right\|_{L_{v}^{p}\left(\mathbb{R}^{n}\right)}^{(2 b+k-1) /(1+k+2(b-a))} .
$$

In particular, under the assumptions stated above, we have

$$
\left\|\nabla^{k} f\right\|_{L_{w}^{q}\left(\mathbb{R}^{n}\right)} \leq C\|f\|_{L_{v}^{p}\left(\mathbb{R}^{n}\right)}^{1-(2 a+k-1) /(1+k)}\left\|\nabla^{k+1} f\right\|_{L_{v}^{p}\left(\mathbb{R}^{n}\right)}^{(2 a+k-1) /(1+k)} .
$$

These theorems have some interesting corollaries.

Corollary 1.4. Let $1 \leq p \leq q<\infty$, and let $\mathscr{D}, W, v$, and $w$ be as in Theorem 1.2 such that

$$
\left\|f-f_{Q}\right\|_{L_{w}^{q}(Q)} \leq A\|\nabla f\|_{L_{v}^{p}(Q)}
$$

for all $Q \in W$ and $f \in \Lambda^{1}(\mathscr{D})$. Then $E_{v, k+1}^{p}(\mathscr{D}) \subset E_{w, k}^{q}(\mathscr{D})$ for all $k \in \mathbb{N}$.

Corollary 1.5. Let $\mathscr{D}$ and $W$ be as in Theorem 1.2. Suppose $1 \leq p \leq q<\infty$ and $w$ is a doubling weight such that

$$
\left\|f-f_{Q}\right\|_{L_{w}^{p}(Q)} \leq A\|\nabla f\|_{L_{w}^{p}(Q)}
$$

for all $Q \in W$ and $f \in \Lambda^{1}(\mathscr{D})$. Then $f \in E_{w, k}^{p}(\mathscr{D})$ if and only if $f \in$ $L_{w, k}^{p}(\mathscr{D})$.

Note that when $w \in A_{p}$ and $\mathscr{D}$ is a bounded $(\varepsilon, \infty)$ domain, it has been obtained in [9] that $E_{w, k}^{p}(\mathscr{D})=L_{w, k}^{p}(\mathscr{D})$.

Corollary 1.6. Let $1 \leq p \leq q<\infty, v \in A_{p}$, and $w$ be a doubling weight such that (1.2) holds. Let $i, k \in \mathbb{N}, 1 \leq i<k$, and $-i<a^{\prime}<k-i$. Then

$$
\left\|\nabla^{i} f\right\|_{L_{w}^{q}\left(\mathbb{R}^{n}\right)} \leq C\|f\|_{L_{v}^{p}\left(\mathbb{R}^{n}\right)}^{1-\left(a^{\prime}+i\right) / k}\left\|\nabla^{k} f\right\|_{L_{v}^{p}\left(\mathbb{R}^{n}\right)}^{\left.(a)^{\prime}+i\right) / k}
$$

for all $f \in \Lambda^{k}\left(\mathbb{R}^{n}\right)$ and $\left\|\nabla^{k} f\right\|_{L_{v}^{q}\left(\mathbb{R}^{n}\right)} \neq 0$ if and only if

$$
l(Q)^{a^{\prime}} w(Q)^{1 / q} \leq C v(Q)^{1 / p}
$$

for all cubes $Q$.

Note that when $p>1, i=1$, and $k=2$, Corollary 1.6 is first obtained by Gutierrez and Wheeden [18].

Finally, similar to Theorems 1.8 and 1.9 in [9], we could apply the extension theorems in [9] to get the following two corollaries. 
Corollary 1.7. Let $1 \leq p \leq q<\infty, v \in A_{p}$, and let $\mathscr{D}$ be a bounded $(\varepsilon, \infty)$ domain. Let $i, k \in \mathbb{N}$ such that $1 \leq i<k$. Let $w$ be a doubling weight such that (1.2) and (1.4) hold for $-i<\bar{a}^{\prime}<k-i$. If $f \in L_{v, k}^{p}(\mathscr{D})$ and there exists a cube $Q$ in $\mathscr{D}$ such that $f=0$ on $Q$ and $\nabla^{k} f \neq 0$ a.e. on $\mathscr{D}$, then

$$
\left\|\nabla^{i} f\right\|_{L_{w}^{q}(\mathscr{D})} \leq C\|f\|_{L_{v}^{p}(\mathscr{D})}^{\left(k-a^{\prime}-i\right) / k}\left\|\nabla^{k} f\right\|_{L_{v}^{p}(\mathscr{D})}^{\left(a^{\prime}+i\right) / k}
$$

where $C$ depends only on $\mathscr{D}, w, v, k, n, p, q$, and $Q$.

Corollary 1.8. Let $\mathscr{D}$ be an unbounded $(\varepsilon, \infty)$ domain, and let $v, w, p, q, i$, and $k$ be as in the preceding corollary. If $f \in L_{v, k}^{p}(\mathscr{D})$ and $\nabla^{k} f \neq 0$ a.e. on $\mathscr{D}$, then

$$
\left\|\nabla^{i} f\right\|_{L_{w}^{q}(\mathscr{D})} \leq C\|f\|_{L_{v}^{p}(\mathscr{D})}^{\left(k-a^{\prime}-i\right) / k}\left\|\nabla^{k} f\right\|_{L_{v}^{p}(\mathscr{D})}^{\left(a^{\prime}+i\right) / k}
$$

where $C$ depends only on $\varepsilon, p, q, v, w, k$, and $n$.

Remark 1.9. (a) Let $\mathscr{D} \subset \mathscr{F}(\sigma, N)$ for some $\sigma, N \geq 1$ and $M \subset \partial \mathscr{D}$ (the boundary of $\mathscr{D})$. Suppose $w(x)=\operatorname{dist}(x, M)=\inf _{y \in M}|x-y|$. Let $W$ be a covering of $\mathscr{D}$ that satisfies the chain condition. Let $\alpha \in \mathbb{R}$. Then it is clear that if $1 \leq p \leq q<\infty$, then

$$
\left\|f-f_{Q}\right\|_{L_{w^{\alpha}}^{q}(Q)} \leq C l(Q)\|\nabla f\|_{L_{w^{\alpha}}^{q}(Q)},
$$

and indeed, when $1-\left(\frac{n}{p}-\frac{n}{q}\right) \geq 0$,

$$
\left\|f-f_{Q}\right\|_{L_{w^{\alpha}}^{q}(Q)} \leq C l(Q)^{1-(n / p-n / q)} \operatorname{dist}(Q, M)^{\alpha / q-\beta / p}\|\nabla f\|_{L_{w^{\beta}}^{p}(Q)}
$$

for $f \in \Lambda^{1}\left(\mathbb{R}^{n}\right)$ and $Q \in W$ with $C$ depending only on $\sigma, N, n, p, \alpha, \beta$, and $q$. These estimates can easily be obtained by the fact that $w$ is comparable to $\operatorname{dist}(Q, M)$ on $Q$ and the unweighted Poincare type estimate.

We can now apply Theorem 1.2 to conclude that when $w^{\alpha}$ is doubling,

$$
\left\|\nabla^{k} f\right\|_{L_{w^{\alpha}}^{q}(\mathscr{D})} \leq C\|f\|_{L^{1}\left(Q_{0}\right)}+C\left\|\nabla^{k+1} f\right\|_{L^{1}\left(Q_{0}\right)}+C\left\|\nabla^{k+1} f\right\|_{L_{w^{\beta}}^{p}(\mathscr{D})}
$$

provided $1-\left(\frac{1}{p}-\frac{1}{q}\right) n+\frac{\alpha}{q}-\frac{\beta}{p} \geq 0$ and $1-\left(\frac{n}{p}-\frac{n}{q}\right) \geq 0$ with $C$ depending only on $\sigma, N, n, p, \alpha, \beta$, and $q$. Hence, for all $k \in \mathbb{N}, E_{w^{\beta}, k+1}^{p}(\mathscr{D}) \subset E_{w^{\alpha}, k}^{p}(\mathscr{D})$ for such $p, q, \alpha$, and $\beta$. Moreover, if $p=q$ and $\alpha=\beta$, we have $E_{w^{\alpha}, k}^{p}(\mathscr{D})=$ $L_{w^{\alpha}, k}^{p}(\mathscr{D})$.

(b) Furthermore, if $w(x)=s(\operatorname{dist}(x, M))$ where $s$ is a positive and continuous function on the positive real numbers that satisfies certain properties described in Kufner [21], a similar conclusion can be obtained by Theorem 1.2 if we know that $w$ is doubling.

(c) We do not know exactly when the weights $w$ defined as above are doubling. However, in the case that $M$ is just a finite subset of $\partial \mathscr{D}$, it is easy to see that $\operatorname{dist}(x, M)^{\alpha}$ is doubling if and only if $\alpha>-n$.

\section{Preliminaries}

In what follows, $C$ denotes various positive constants. They may differ even in the same string of estimates. Moreover, sometimes, we will use $C(\alpha, \beta, \ldots)$ instead of $C$ to emphasize that the constant is depending on $\alpha, \beta, \ldots$.

Since one of our main tools will be a projection of functions into polynomials, first let us state an inequality on polynomials. 
Theorem 2.1. Let $F, Q$ be cubes such that $F \subset Q$ and $|F|>\gamma|Q|$. If $w$ is a doubling weight, $1 \leq q<\infty$, and $p$ is a polynomial of degree $m$, then

$$
\|p\|_{L_{w}^{q}(E)} \leq C(\gamma, m, n, w)\left(\frac{w(E)}{w(F)}\right)^{1 / q}\|p\|_{L_{w}^{q}(F)}
$$

for all measurable sets $E \subset Q$.

This theorem is just a consequence of the following two lemmas.

Lemma 2.2 [29, Chapter 3, Lemma 7]. If $w$ is a doubling measure and $m$ is a positive integer, then there exists $s_{0}(n, m, w)$ such that if $s<s_{0}$, then for all cubes $Q, \lambda>0$ such that

$$
w(\{x \in Q:|p(x)|>\lambda\}) \leq s w(Q)
$$

we have $\sup _{x \in Q}|p(x)| \leq C \lambda$, where $p$ is any polynomial of degree $m$ and $C$ is a constant independent of $\lambda, Q$, and $p$.

It follows from Chebyshev's inequality and this lemma that given $m$ and a polynomial $p$ of degree $m$,

$$
\|p\|_{L^{\infty}(Q)} \leq \frac{C}{w(Q)}\|p\|_{L_{w}^{1}(Q)}
$$

with $C$ independent of $Q$ and $p$.

Lemma 2.3 [9, Theorem 2.2]. Let $Q$ be a cube, and let $E$ be a measurable set in $Q$ with $|E|>\gamma|Q|$. If $p$ is a polynomial of degree $m$, then

$$
\|p\|_{L^{\infty}(E)} \geq C(\gamma, m)\|p\|_{L^{\infty}(Q)} \text {. }
$$

Next, let us state Markov's inequality; see, for example, [1].

Theorem 2.4. Let $p$ be any polynomial of order less than $k$. Then there exists a constant $C$ depending only on $k$ and the dimension $n$ such that

$$
\|\nabla p\|_{L^{\infty}(Q)} \leq C l(Q)^{-1}\|p\|_{L^{\infty}(Q)} \text { for all cubes } Q \text { in } \mathbb{R}^{n} .
$$

Finally, the following is now a consequence of Markov's inequality and Lemma 2.2.

Theorem 2.5. Let $p$ be a polynomial of order less than $k$ and $1 \leq q \leq \infty$. If $w$ is doubling, then

$$
\|\nabla p\|_{L_{w}^{q}(Q)} \leq C l(Q)^{-1}\|p\|_{L_{w}^{q}(Q)}
$$

for all cubes $Q$ in $\mathbb{R}^{n}$, where $C$ depends only on $k, w, q$, and $n$.

Now let us state a theorem from [12].

Theorem 2.6. Let $\sigma, N \geq 1,1 \leq p \leq q<\infty, k \in \mathbb{N}$, and $\mathscr{D} \in \mathscr{F}(\sigma, N)$, and let $f, g$ be measurable functions defined on $\mathscr{D}$. Also, let $v$ be a weight, and let $w$ be a doubling weight. Suppose that for each cube $Q$ with $\sigma Q \subset \mathscr{D}$, there exists a polynomial $P(f, Q)$ of degree $k$ such that

$$
\|f-P(f, Q)\|_{L_{w}^{q}(Q)} \leq A\|g\|_{L_{v}^{p}(\sigma Q)}
$$

with $A$ independent of $Q$. Then there exists a polynomial $P(f, \mathscr{D})$ of degree $k$ such that

$$
\|f-P(f, \mathscr{D})\|_{L_{w}^{q}(\mathscr{D})} \leq C A\|g\|_{L_{v}^{p}(\mathscr{D})}
$$


where $C$ depends only on $n, q, w, \sigma, k$, and $N$. Moreover, we can take $P(f, \mathscr{D})=P\left(f, Q_{0}\right)$ where $Q_{0}$ is the 'central' cube in $\mathscr{D}$.

Let $\mathscr{P}_{k}$ be the collection of all polynomials with degree $<k$ on $\mathbb{R}^{n}$. Now, let us state a theorem concerning the projection of function into polynomials.

Theorem 2.7. Let $\mathscr{D}$ be an open set. For each $k \in \mathbb{N}$ and cubes $Q \subset \mathscr{D}$, there exists a projection $\pi_{k}(Q): \Lambda^{k}(\mathscr{D}) \rightarrow \mathscr{P}_{k}$ such that

$$
\underset{x \in Q}{\operatorname{ess} \sup }\left|\pi_{k}(Q) f(x)\right| \leq C l(Q)^{-n}\|f\|_{L^{1}(Q)}
$$

with $C$ independent of $f$ and $Q$. Moreover, $\pi_{k}(Q)$ is linear and $\pi_{k}(Q) p=p$ for all $p \in \mathscr{P}_{k}$.

For the proof, please refer to [9] or [10].

Finally, let us state the weighted Poincaré inequality for $A_{p}$ weights. For the proof, see [9].

Theorem 2.8. If $1 \leq p<\infty$, and $v \in A_{p}$, then

$$
\left\|f-f_{Q}\right\|_{L_{v}^{p}(Q)} \leq C l(Q)\|\nabla f\|_{L_{v}^{p}(Q)}
$$

for all cubes $Q$ and $f \in \Lambda^{1}\left(\mathbb{R}^{n}\right)$ where $C$ depends only on $p, v$, and $n$.

\section{Proof of MAIN Results}

Proof of Theorem 1.2. First let us fix $f \in \Lambda^{2}(\mathscr{D})$ such that $\left|\nabla^{2} f\right| \in L_{v}^{p}(\mathscr{D})$. Next, we let $P_{Q_{0}} f$ be the polynomial of degree 1 such that $\int_{Q_{0}} D^{\alpha}\left(f-P_{Q_{0}} f\right) d x=$ 0 for all $|\alpha| \leq 1$. Now, by Theorem 2.6,

$$
\left\|\nabla\left(f-P_{Q_{0}} f\right)\right\|_{L_{w}^{q}(\mathscr{D})} \leq C A_{2}\left\|\nabla^{2} f\right\|_{L_{v}^{p}(\mathscr{D})},
$$

where $A_{2}=\sup _{Q \in W} A(Q)$. Next, let us note that

$$
\begin{aligned}
\left\|\nabla P_{Q_{0}} f\right\|_{L_{w}^{q}(\mathscr{D}) \leq} \leq & C\left\|\nabla P_{Q_{0}} f\right\|_{L_{w}^{q}\left(Q_{0}\right)} \quad \text { (by Theorem 2.1) } \\
\leq & C\left\|\nabla\left(P_{Q_{0}} f-\pi_{2}\left(Q_{0}\right) f\right)\right\|_{L_{w}^{q}\left(Q_{0}\right)}+\left\|\nabla \pi_{2}\left(Q_{0}\right) f\right\|_{L_{w}^{q}\left(Q_{0}\right)} \\
\leq & C l\left(Q_{0}\right)^{-1}\left\|P_{Q_{0}} f-\pi_{2}\left(Q_{0}\right) f\right\|_{L_{w}^{q}\left(Q_{0}\right)} \\
& +C l\left(Q_{0}\right)^{-1}\left\|\pi_{2}\left(Q_{0}\right) f\right\|_{L_{w}^{q}\left(Q_{0}\right)} \quad \text { by Theorem 2.5) } \\
\leq & C l\left(Q_{0}\right)^{-1-n} w\left(Q_{0}\right)^{1 / q}\left\|f-P_{Q_{0}} f\right\|_{L^{1}\left(Q_{0}\right)} \\
& +C l\left(Q_{0}\right)^{-1-n} w\left(Q_{0}\right)^{1 / q}\|f\|_{L^{1}\left(Q_{0}\right)} \quad \text { (by Theorem 2.7) } \\
\leq & C l\left(Q_{0}\right)^{1-n} w\left(Q_{0}\right)^{1 / q}\left\|\nabla^{2} f\right\|_{L^{1}\left(Q_{0}\right)}+C l\left(Q_{0}\right)^{-1-n} w\left(Q_{0}\right)^{1 / q}\|f\|_{L^{1}\left(Q_{0}\right)}
\end{aligned}
$$

by the nonweighted Poincaré inequality. Hence

$$
\begin{aligned}
\|\nabla f\|_{L_{w}^{q}(\mathscr{D})} \leq & C\left\|\nabla P_{Q_{0}} f\right\|_{L_{w}^{q}(\mathscr{D})}+C A_{2}\left\|\nabla^{2} f\right\|_{L_{v}^{p}(\mathscr{D})} \\
\leq & C l\left(Q_{0}\right)^{1-n} w\left(Q_{0}\right)^{1 / q}\left\|\nabla^{2} f\right\|_{L^{1}\left(Q_{0}\right)} \\
& +C l\left(Q_{0}\right)^{-1-n} w\left(Q_{0}\right)^{1 / q}\|f\|_{L^{1}\left(Q_{0}\right)}+C A_{2}\left\|\nabla^{2} f\right\|_{L_{v}^{p}(\mathscr{D})} .
\end{aligned}
$$


Proof of Theorem 1.3. Similar to the proof as above, for any cube $Q$, we have

$$
\begin{aligned}
\left\|\nabla^{k} f\right\|_{L_{w}^{q}(Q) \leq} & C w(Q)^{1 / q} l(Q)^{-k-n}\|f\|_{L^{1}(Q)}+C w(Q)^{1 / q} l(Q)^{1-n}\left\|\nabla^{k+1} f\right\|_{L^{1}(Q)} \\
& +C w(Q)^{1 / q} v^{\prime}(Q)^{1 / p^{\prime}} l(Q)^{-n+1}\left\|\nabla^{k+1} f\right\|_{L_{v}^{p}(Q)} \\
\leq & C w(Q)^{1 / q} l(Q)^{-k-n} v_{0}^{\prime}(Q)^{1 / p_{0}^{\prime}}\|f\|_{L_{v_{0}}^{p_{0}}(Q)} \\
& +C w(Q)^{1 / q} v^{\prime}(Q)^{1 / p^{\prime}} l(Q)^{-n+1}\left\|\nabla^{k+1} f\right\|_{L_{v}^{p}(Q)}
\end{aligned}
$$

by Hölder's inequality. Next, if (1.3) holds, then for all cubes $Q$ we have

$$
\left\|\nabla^{k} f\right\|_{L_{w}^{q}(Q)} \leq C l(Q)^{-2 b-k+1}\|f\|_{L_{v_{0}}^{p_{0}}(Q)}+C l(Q)^{2-2 a}\left\|\nabla^{k+1} f\right\|_{L_{v}^{p}(Q)} .
$$

Now, for all $\varepsilon>0$, we will cover $\mathbb{R}^{n}$ by cubes of length $\varepsilon$. Let $W^{\prime}$ be any nonoverlapping cover of cubes with length $\varepsilon$. Summing up the cubes, we have

$$
\|\nabla f\|_{L_{w}^{q}\left(\mathbb{R}^{n}\right)}^{q} \leq C \varepsilon^{(-2 b-k+1) q} \sum_{Q \in W^{\prime}}\|f\|_{L_{v_{0}}^{p_{0}}(Q)}^{q}+C \varepsilon^{(2-2 a) q} \sum_{Q \in W^{\prime}}\left\|\nabla^{k+1} f\right\|_{L_{v}^{p}(Q)}^{q} .
$$

Thus

$$
\begin{aligned}
\left\|\nabla^{k} f\right\|_{L_{w}^{q}\left(\mathbb{R}^{n}\right)} \leq & C \varepsilon^{-2 b-k+1}\left(\sum_{Q \in W^{\prime}}\|f\|_{L_{v_{0}}^{p_{0}}(Q)}^{p_{0}}\right)^{1 / p_{0}} \\
& +C \varepsilon^{(2-2 a)}\left(\sum_{Q \in W^{\prime}}\left\|\nabla^{k+1} f\right\|_{L_{v}^{p}(Q)}^{p}\right)^{1 / p}
\end{aligned}
$$

since $q \geq p_{0}, p$. Hence,

$$
\|\nabla f\|_{L_{w}^{q}\left(\mathbb{R}^{n}\right)} \leq C \varepsilon^{-2 b-k+1}\|f\|_{L_{v_{0}}^{p_{0}}\left(\mathbb{R}^{n}\right)}+C \varepsilon^{(2-2 a)}\left\|\nabla^{k+1} f\right\|_{L_{v}^{p}\left(\mathbb{R}^{n}\right)} .
$$

To complete the proof just choose $\varepsilon=\left(\|f\|_{L_{v_{0}}^{p_{0}}\left(\mathbb{R}^{n}\right)} /\left\|\nabla^{k+1} f\right\|_{L_{v}^{p}\left(\mathbb{R}^{n}\right)}\right)^{1 /(1+k+2(b-a))}$.

Next, let us note that Corollaries 1.4 and 1.5 are just immediate consequences of Theorem 1.2.

Proof of Corollary 1.6. First note that by similar arguments as above, for any cube $Q$ and $f \in L_{v, k}^{p}\left(\mathbb{R}^{n}\right)$, let $P_{Q} f$ be the unique polynomial of degree $<k$ such that $\int_{Q} D^{\alpha}\left(f-P_{Q} f\right) d x=0$ for all $|\alpha|<k$, we have

$$
\begin{aligned}
\left\|\nabla^{i} P_{Q} f\right\|_{L_{w}^{q}(Q) \leq} & C w(Q)^{1 / q} l(Q)^{-i-n}\|f\|_{L^{1}(Q)}+C w(Q)^{1 / q} l(Q)^{-n+k-i}\left\|\nabla^{k} f\right\|_{L^{1}(Q)} \\
\leq & C w(Q)^{1 / q} l(Q)^{-i} v(Q)^{-1 / p}\|f\|_{L_{v}^{p}(Q)} \\
& +C w(Q)^{1 / q} v(Q)^{-1 / p} l(Q)^{k-i}\left\|\nabla^{k} f\right\|_{L_{v}^{p}(Q)}
\end{aligned}
$$

since $v \in A_{p}$. Also,

$$
\begin{aligned}
\left\|\nabla^{i}\left(f-P_{Q} f\right)\right\|_{L_{w}^{q}(Q)} & \leq C l(Q) w(Q)^{1 / q} v(Q)^{-1 / p}\left\|\nabla^{i+1}\left(f-P_{Q} f\right)\right\|_{L_{v}^{p}(Q)} \\
& \leq C l(Q)^{k-i} w(Q)^{1 / q} v(Q)^{-1 / p}\left\|\nabla^{k} f\right\|_{L_{v}^{p}(Q)}
\end{aligned}
$$

by Theorem 2.8 . Hence by the triangle inequality and (1.4),

$$
\begin{aligned}
\left\|\nabla^{i} f\right\|_{L_{w}^{q}(Q)} & \leq\left\|\nabla^{i}\left(f-P_{Q} f\right)\right\|_{L_{w}^{q}(Q)}+\left\|\nabla^{i} P_{Q} f\right\|_{L_{w}^{q}(Q)} \\
& \leq C l(Q)^{-a^{\prime}-i}\|f\|_{L_{v}^{p}(Q)}+C l(Q)^{-a^{\prime}+k-i}\left\|\nabla^{k} f\right\|_{L_{v}^{p}(Q)} .
\end{aligned}
$$


We can now just follow as before to show

$$
\left\|\nabla^{i} f\right\|_{L_{w}^{q}\left(\mathbf{R}^{n}\right)} \leq C\|f\|_{L_{v}^{p}\left(\mathbb{R}^{n}\right)}^{1-\left(a^{\prime}+i\right) / k}\left\|\nabla^{k} f\right\|_{L_{v}^{p}\left(\mathbf{R}^{n}\right)}^{\left(a^{\prime}+i\right) / k}
$$

for all $f \in L_{v, k}^{p}\left(\mathbb{R}^{n}\right)$ with $\left\|\nabla^{k} f\right\|_{L_{v}^{q}\left(\mathbb{R}^{n}\right)} \neq 0$. Finally, the reader can refer to the proof of Theorem 5 in Gutierrez and Wheeden [18] for the converse.

Finally, let us note that the proof of Corollaries 1.7 and 1.8 are almost identical to the proof of Theorems 1.8 and 1.9 in [9] with the help of Corollary 1.6.

\section{REFERENCES}

1. Bogdan Bojarski, Remarks on Sobolev imbedding inequalities, Complex Analysis, Lecture Notes in Math., vol. 1351, Springer-Verlag, New York, 1989, pp. 52-68.

2. Richard Adams, Sobolev spaces, Academic Press, New York, 1975.

3. R. C. Brown and D. B. Hilton, Sufficient conditions for weighted inequalities of sum form, J. Math. Anal. Appl. 112 (1985), 563-578.

4. $\ldots$, Weighted interpolation inequalities of sum and product form in $\mathbb{R}^{n}$, Proc. London Math. Soc. (3) 56 (1988), 261-280.

5. — Weighted interpolation inequalities and embeddings in $\mathbb{R}^{n}$, Canad. J. Math. 62 (1990), 959-980.

6. C. Carton-Lebrun and H. P. Heinig, Weighted norm inequalities involving gradients, Proc. Roy. Soc. Edinburgh Sect. A 112 (1989), 331-341.

7. Sagun Chanillo and Richard L. Wheeden, Poincaré inequalities for a class of non- $A_{p}$ weights, Indiana Math. J. 41 (1992), 605-623.

8. Filippo Chiarenza and Michelle Frasca, $A$ note on a weighted Sobolev inequality, Proc. Amer. Math. Soc. 93 (1985), 703-704.

9. Seng-Kee Chua, Extension theorems on weighted Sobolev spaces, Indiana Math. J. 41 (1992), 1027-1076.

10. __ Extension and restriction theorems on weighted Sobolev spaces, Ph.D. Thesis, Rutgers University, 1991.

11. __ Some remarks on extension theorems for weighted Sobolev spaces, Illinois J. Math. (to appear).

12. _ Weighted Sobolev's inequalities on domains satisfying the chain condition, Proc. Amer. Math. Soc. 117 (1993), 449-457.

13.

14. D. E. Edmunds, Alois Kufner, and Jiong Sun, Extension of functions in weighted Sobolev spaces, Rend. Accad. Naz. Sci. XL Mem. Mat. (5) 16 (1990), 327-339.

15. Eugene B. Fabes, Carlos E. Kenig, and Raul P. Serapioni, The local regularity of solutions of degenerate elliptic equations, Comm. Partial Differential Equations 7 (1982), 77-116.

16. David Gilbarg and Neil S. Trudinger, Elliptic partial differential equations of second order, Springer-Verlag, New York, 1983.

17. V. M. Gol'dshtein and Yu. G. Reshetnyak, Quasiconformal mappings and Sobolev spaces, Kluwer, Dordrecht, 1990.

18. Cristian E. Gutierrez and Richard L. Wheeden, Sobolev interpolation inequalities with weights, Trans. Amer. Math. Soc. 323 (1991), 263-281.

19. T. Iwaniec and C. A. Nolder, Hardy-Littlewood inequality for quasiregular mappings in certain domains in $\mathbb{R}^{n}$, Ann. Acad. Sci. Fenn. Ser. A I Math. 10 (1985), 267-282.

20. Peter Jones, Quasiconformal mappings and extendability of functions in Sobolev spaces, Acta Math. 147 (1981), 71-88.

21. Alois Kufner, Weighted Sobolev spaces, Wiley, New York, 1985. 
22. __ How to define reasonable Sobolev spaces, Comment. Math. Univ. Carolin. 25 (1984), 537-554.

23. Olli Martio and J. Sarvas, Injectivity theorems in plane and space, Ann. Acad. Sci. Fenn. Ser. A I Math. 4 (1979), 383-401.

24. Vladimir G. Maz'ja, Sobolev spaces, Springer-Verlag, New York, 1985.

25. Benjamin Muckenhoupt, Weighted norm inequalities for the Hardy maximal function, Trans. Amer. Math. Soc. 165 (1972), 207-226.

26. Carlos Pérez, Two weighted norm inequalities for Riesz Potentials and uniform $L^{p}$-weighted Sobolev inequalities, Indiana Math. J. 39 (1990), 31-44.

27. Eric Sawyer and Richard L. Wheeden, Weighted inequalities for fractional integrals on Euclidean and homogeneous spaces, Amer. J. Math. 114 (1992), 813-874.

28. Elias M. Stein, Singular integrals and differentiability properties of functions, Princeton Univ. Press, Princeton, NJ, 1970.

29. Jan-Olov Stromberg and Alberto Torchinsky, Weighted Hardy spaces, Lecture Notes in Math., vol. 1381, Springer, New York, 1989.

30. Alberto Torchinsky, Real-variable methods in harmonic analysis, Academic Press, New York, 1986.

31. Richard L. Wheeden and Antoni Zygmund, Measure and integral, Marcel Dekker, New York, 1977.

Department of Mathematics, National University of Singapore, 10 Kent Ridge Road, SINGAPORE 0511, SINGAPORE

E-mail address: matcsk@nusunix.nus.sg 